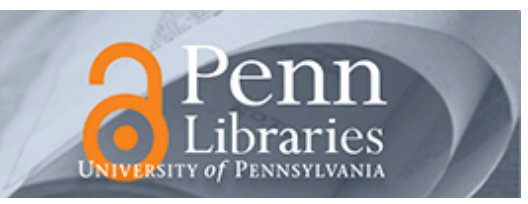

University of Pennsylvania

ScholarlyCommons

June 2002

\title{
A Time-Scale Decomposition Approach to Adaptive Explicit Congestion Notification (ECN) Marking
}

\author{
Srisankar S. Kunniyur \\ University of Pennsylvania, kunniyur@seas.upenn.edu \\ R. Srikant \\ University of Illinois
}

Follow this and additional works at: https://repository.upenn.edu/ese_papers

\section{Recommended Citation}

Srisankar S. Kunniyur and R. Srikant, "A Time-Scale Decomposition Approach to Adaptive Explicit Congestion Notification (ECN) Marking", . June 2002.

Copyright 2002 IEEE. Reprinted from IEEE Transactions on Automatic Control, Volume 47, Issue 6, June 2002, pages 882-894.

Publisher URL: http://ieeexplore.ieee.org/xpl/tocresult.jsp?isNumber=21741\&puNumber=9

This material is posted here with permission of the IEEE. Such permission of the IEEE does not in any way imply IEEE endorsement of any of the University of Pennsylvania's products or services. Internal or personal use of this material is permitted. However, permission to reprint/republish this material for advertising or promotional purposes or for creating new collective works for resale or redistribution must be obtained from the IEEE by writing to pubs-permissions@ieee.org. By choosing to view this document, you agree to all provisions of the copyright laws protecting it.

This paper is posted at ScholarlyCommons. https://repository.upenn.edu/ese_papers/51

For more information, please contact repository@pobox.upenn.edu. 


\title{
A Time-Scale Decomposition Approach to Adaptive Explicit Congestion Notification (ECN) Marking
}

\begin{abstract}
Fair resource allocation in high-speed networks, such as the Internet, can be viewed as a constrained optimization program. Kelly et al. have shown that an unconstrained penalty function formulation of this problem can be used to design congestion controllers that are stable. In this paper, we examine the question of providing feedback from the network such that the congestion controllers derived from the penalty function formulation lead to the solution of the original unconstrained problem. This can be viewed as the decentralized design of explicit congestion notification (ECN) marking rates at each node in the Internet to ensure global loss-free operation of a fluid model of the network. We then look at the stability of such a scheme using a time-scale decomposition of the system. This results in two seperate systems which are stable individually, and we show that under certain assumptions the entire system is semiglobally stable and converges fast to the equilibrium point exponentially.
\end{abstract}

\section{Keywords}

Adaptive virtual queue, congestion control, explicit congestion notification (ECN) marking, Internet, singular perturbations

\section{Comments}

Copyright 2002 IEEE. Reprinted from IEEE Transactions on Automatic Control, Volume 47, Issue 6, June 2002, pages 882-894.

Publisher URL: http://ieeexplore.ieee.org/xpl/tocresult.jsp?isNumber=21741\&puNumber=9

This material is posted here with permission of the IEEE. Such permission of the IEEE does not in any way imply IEEE endorsement of any of the University of Pennsylvania's products or services. Internal or personal use of this material is permitted. However, permission to reprint/republish this material for advertising or promotional purposes or for creating new collective works for resale or redistribution must be obtained from the IEEE by writing to pubs-permissions@ieee.org. By choosing to view this document, you agree to all provisions of the copyright laws protecting it. 


\title{
A Time-Scale Decomposition Approach to Adaptive Explicit Congestion Notification (ECN) Marking
}

\author{
Srisankar Kunniyur and R. Srikant, Senior Member, IEEE
}

\begin{abstract}
Fair resource allocation in high-speed networks, such as the Internet, can be viewed as a constrained optimization program. Kelly et al. have shown that an unconstrained penalty function formulation of this problem can be used to design congestion controllers that are stable. In this paper, we examine the question of providing feedback from the network such that the congestion controllers derived from the penalty function formulation lead to the solution of the original unconstrained problem. This can be viewed as the decentralized design of explicit congestion notification (ECN) marking rates at each node in the Internet to ensure global loss-free operation of a fluid model of the network. We then look at the stability of such a scheme using a time-scale decomposition of the system. This results in two seperate systems which are stable individually, and we show that under certain assumptions the entire system is semiglobally stable and converges fast to the equilibrium point exponentially.
\end{abstract}

Index Terms-Adaptive virtual queue, congestion control, explicit congestion notification (ECN) marking, Internet, singular perturbations.

\section{INTRODUCTION}

$\mathbf{R}$ ECENTLY, there has been an explosive growth in the development of new applications that use the Internet. As a result, congestion and packet loss resulting from congestion has become a big problem. To offer multimedia services over the Internet, some of which may require low-loss, low-delay operation, it is desirable to provide explicit congestion notification (ECN) [1]-[3]. Therefore, there has been a surge of interest in designing best-effort service networks that can deliver low-loss, low-delay service by encouraging the users to adapt to network congestion using minimal information from the network in the form of ECN marks [1], [4]-[6].

In a fluid model, the problem of fair resource allocation to heterogeneous users over a heterogeneous network can be posed as a convex program [4]-[6]. The design of congestion controllers then follows by considering either a penalty function formulation of the problem [4], [6] or a dual formulation of the problem [5]. For the dual problem, it has been shown in [5], that a simple adaptive algorithm to estimate the shadow prices at each node based only on total flow measurements at each node, together with appropriate congestion control algorithms for the users, can

Manuscript received September 13, 2000; revised June 7, 2001 and November 15, 2001. Recommended by the Guest Editors W. Gong and T. Başar. This work was supported by the National Science Foundation under Grants NCR-9701525 and ANI-9813710

S. Kunniyur is with the Department of Electrical Engineering, University of Pennsylvania, Philadelphia, PA 19104 USA (e-mail: kunniyur@ee.upenn.edu).

R. Srikant is with the Coordinated Science Laboratory, Department of General Engineering, University of Illinois, Urbana, IL 61801-2307 USA (e-mail: rsrikant@uiuc.edu).

Publisher Item Identifier S 0018-9286(02)05496-X. lead to the optimal solution of the convex program. Further, it has been shown in [7] that the shadow prices can be conveyed to the sources using a single-bit mark in each packet.

In this paper, we study the adaptive design of the marking rates at each node to drive network to the optimal fluid operating point in the case where the congestion controllers are derived using a penalty function formulation. The penalty function formulation is appealing due to the close connection between the congestion controller so derived and the well-known TCP protocol [6]. We seek an adaptive algorithm that modifies the fraction of packets marked (i.e., the marking probability in a model that treats packets as discrete entities) at each node such that the total fluid entering each link is less than or equal to the capacity of the link (i.e., near loss-free operation) while maximizing the sum of the utilities of all the users of the network (socially optimal operation). By "capacity of the link," we mean the maximum arrival rate at the link that will maintain a near loss-free operation. For example, in a practical network with variable packet sizes, round-trip delays and buffers, this maximum arrival rate may be as high as $98 \%$ of the actual capacity of the link. The motivation for allowing high utilization comes from many-sources large-deviation results [8] which suggest that low-loss operation is possible even with high utilization when the number of users and the capacity of the network are large.

For certain constrained nonlinear programming problems, it is well-known that penalty functions can be designed such that the solution of the unconstrained problem with these penalty functions leads to the solution of the original, constrained problem. Such penalty functions are called exact penalty functions [9, Sec. 4.3]. Thus, the adaptive algorithm in this paper can be viewed as a distributed algorithm for obtaining exact penalty functions, without each node having any direct information about the topology of the rest of the network.

The key features of our adaptive algorithm are summarized as follows.

1) Each node chooses an algorithm according to which it marks packets.

2) The marking algorithm at each node could be different, i.e., the nodes need not agree on the same algorithm.

3) Each node's marking algorithm is parameterized by a quantity, called the node's "virtual capacity." The fraction of marked packets at the node is a function of the total arrival rate at the node and the virtual capacity of the node.

4) The fraction of packets that are marked at a node can be any function of the total arrival rate at the node and the virtual capacity of the node as long as it satisfies certain 
reasonable properties: it should an increasing function of the total arrival rate and a decreasing function of the virtual capacity. More technical conditions are discussed in the later sections.

5) Each node uses an algorithm to adapt its virtual capacity to drive the fluid model of the network toward loss-free, socially optimal operation. The adaptive algorithm at a node uses only the total flow rate at that node, i.e., no per-flow measurements are required.

With this adaptation at the links, the dynamics of the network can be thought of as evolving at two timescales.

1) The sources implement the congestion-controllers to maximize the sum of the utilities of all the users (minus a penalty function) using the feedback from the links.

2) Each link modifies its marking function so that the total average flow (over some time) is less than or equal to the capacity of the link.

So, there is a natural two-time scale behavior of the system in that the sources adapt quickly while the links adapt more slowly. We can now decompose the system into two parts.

1) The slow system or the reduced system: In this system, we assume that the user rates have converged for a particular virtual capacity at the links and the links then modify their virtual capacities based on these optimum rates. This is the slow model because we assume that the changes at the links are slow enough to make the user rates' converge.

2) The boundary-layer system: Here, we assume that the virtual capacity remains constant, and therefore, the system is characterized by the set of congestion-controllers for the original system with a fixed virtual capacity at each link.

The entire system's response to external stimuli (i.e., initial condition) can now be characterized by the presence of slow and fast transients. Loosely speaking, we can approximate the slow transient by the reduced system and the fast transient is the difference between the full system and the reduced-system [10].

In this paper, we study the stability properties of the entire system which comprises the congestion-controllers of the users and the adaptive marking algorithm at the links. To study the stability of the entire system, we start by studying the reduced-model and the boundary-layer model of the system. The boundary-layer model of the system is shown to be globally asymptotically stable in [4]. In this paper, we will show that the reduced system is stable whenever all the links in the network are fully utilized. We will also show that both the reduced system and the boundary-layer system are semiglobally exponentially stable. By semiglobal exponential stability, we mean that the system is exponentially stable whenever the system is constrained to lie in a compact set. We will then show that under certain assumptions, the entire system is semiglobally stable and converges to the stable point exponentially. In congestion-controlled systems such as the Internet, the transmission rates of all sources are constrained. Thus, the set of allowable initial conditions is compact. Therefore, "semiglobal" is effectively equivalent to global stability in our context. We will also show that the equilibrium point maximizes the sum of the utilities of all the users and also results in a near-loss free operation throughout the network. We use the term near-loss free operation to indicate that the fluid model of the system is loss free. However, the presence of extremely short flows and other randomness in the system will lead to some loss unless one operates the systems at slightly less than full utilization. This is explored further in [11] for the case of fixed virtual capacities.

The rest of this paper is organized as follows. In Section II, we first describe the network model and define the system optimization problem. We will also define and state our assumptions on the marking algorithms at the nodes. In Section III, we prove that the algorithm succeeds in conveying the sum of the shadow prices of each route to the users of the network. We first show the existence of virtual capacities that results in a loss-free network. We will then show that with these virtual capacities, we obtain the system optimum rates. We then show that the algorithm indeed converges to virtual capacities that results in a loss-free, socially optimal operation. We then proceed to prove the stability of the reduced-system. In Section IV, we prove the semiglobal exponential stability of the entire system. Concluding remarks are provided in Section V. For ease of exposition, some of the proofs are presented in the Appendix (Section VII).

\section{SYSTEM MODEL}

We adopt the system model described by Kelly in [12]. Consider a network with a set $\mathcal{L}$ of links and let $C_{l}$ be the capacity of link $l$, for $l \in \mathcal{L}$. Let a route $r$ be a nonempty subset of $\mathcal{L}$, and let $\mathcal{R}$ be the set of possible routes. Set $S_{r l}=1$ if $l \in r$, so that route $r$ traverses link $l$ and set $S_{r l}=0$ otherwise. We will associate an user with each route and hence we will use the term "user" and "route" interchangeably throughout this paper. Let user $r$ generate traffic at rate $x_{r}$. The rate $x_{r}$ is assumed to have a utility $U_{r}\left(x_{r}\right)$ to user $r$. Assume that $U_{r}(\cdot)$ is a continuously differentiable, strictly concave, increasing function in the interval $(0, \infty)$ and we assume that $U_{r}\left(x_{r}\right)$ is unbounded as $x_{r} \rightarrow 0$ to ensure that each user gets some nonzero throughput. Examples of such a function include $\log x_{r}$ and $-1 / x_{r}$. Let $U=\left(U_{r}(\cdot), r \in \mathcal{R}\right), x=\left(x_{r}, r \in \mathcal{R}\right)$ and $C=\left(C_{l}, l \in \mathcal{L}\right)$. The optimal rates for this network can now be obtained by solving the following maximization problem:

$$
\operatorname{SYSTEM}(U, S, C): \quad \max _{\left\{x_{r}\right\}} \sum_{r} \Delta_{r} U_{r}\left(x_{r}\right)
$$

subject to

$$
\begin{gathered}
S^{T} x \leq C \\
x \geq 0
\end{gathered}
$$

where $\Delta_{r}>0$. The above maximization problem has a strictly concave objective function and the maximization is performed over a compact set. Therefore, the above maximization problem has a unique solution. In the rest of the paper, we refer to the solution to the aforementioned maximization problem as the system optimal rates.

Let each link $l$ in the network generate feedback in the form of ECN marks. Assume that the fraction of packets marked is a function of the total arrival rate $\left(\lambda_{l}\right)$ and the virtual capacity 
$\left(\tilde{C}_{l}\right)$ of the link and that the total marks are distributed among the users in proportion to their flow rates. We also assume that the same flow is presented by a user to all links on its route, even though the flow will actually be "thinned" in downstream links due to losses at upstream links (link independence assumption). Therefore, the total flow into any link $l$ is given by $\lambda_{l}=\sum_{r: l \in r} x_{r}$. Let $p_{l}\left(\lambda_{l}, \tilde{C}_{l}\right)$ be the fraction of the total flow that is marked by link $l$. Therefore, if User $r$ has a rate $x_{r}$ going into link $l$, then the total rate at which marks are received by User $r$ from link $l$ is $p_{l}\left(\lambda_{l}, \tilde{C}_{l}\right) x_{r}$. We will make the following assumption regarding the marking functions at each link.

Assumption 1: The marking function at each link $l \in \mathcal{L}, p_{l}(q, s), q \geq 0, s \geq 0$ is assumed to satisfy the following conditions:

i) $0 \leq p_{l}(q, s) \leq 1$ for all $q, s$;

ii) $\lim _{q \rightarrow \infty} p_{l}(q, s)=1$ for all $s$;

iii) $\lim _{s \rightarrow \infty} p_{l}(q, s)=0$ for all $q$;

iv) $\lim _{s \rightarrow 0} p_{l}(q, s)=1$ for all $q$;

v) $p_{l}(q, s)$ is strictly increasing in $q$ and strictly decreasing in $s$;

vi) $p_{l}(q, s)$ is twice continuously differentiable in both its arguments;

vii) For each $l \in \mathcal{L}$, there exists a virtual capacity $\tilde{C}_{l}^{(d)} \geq 0$ which solves

$$
C_{l}=\sum_{r: l \in r} U_{r}^{\prime-1}\left[\frac{\beta}{\Delta_{r}} p_{l}\left(C_{l}, \tilde{C}_{l}\right)\right] .
$$

This assumption states that if we consider each link $l \in \mathcal{L}$ in isolation (by assuming all the other links have infinite capacity), then we can find a virtual capacity $\tilde{C}_{l}^{(d)}$ such that solution $\left(x_{r}^{*}, r \in l\right)$ to the optimization problem

$$
\max _{\left\{x_{r}: r \in l\right\}} \sum_{r: r \in l} \Delta_{r} U_{r}\left(x_{r}\right)-\beta \int_{0}^{\sum_{r: l \in r} x_{r}} p_{l}\left(z, \tilde{C}_{l}\right) d z
$$

satisfies $\sum_{r: r \in l} x_{r}^{*}=C_{l}$. In other words, we assume that the network is provisioned such that each node considered in isolation can choose a virtual capacity to provide loss-free operation.

Now, let each User $r$ employ the congestion-control algorithm,

$$
\dot{x}_{r}=\left(\Delta_{r}-\frac{\beta}{U_{r}^{\prime}\left(x_{r}\right)} \sum_{l: l \in r} p_{l}\left(\lambda_{l}, \tilde{C}_{l}\right)\right) \quad \forall r \in \mathcal{R} .
$$

This is an additive-increase, multiplicative-decrease algorithm wherein the user reduces its rate proportional to the number of marks it receives. From [4], we know that the congestion-control scheme given by (6) converges to the unique optimal solution of the optimization problem given by

$$
\max _{\left\{x_{r}\right\}} \sum_{r} \Delta_{r} U_{r}\left(x_{r}\right)-\beta \sum_{l \in \mathcal{L}} \int_{0}^{\lambda_{l}} p_{l}\left(z, \tilde{C}_{l}\right) d z .
$$

The optimal solution of the optimization problem given by (7) depends upon the virtual capacity at each link. Therefore, by adaptively changing the virtual capacities, we can ensure that the total flow into the link is less than the capacity of the link and thus ensure loss-free operation. We also need to take care that we are not under-utilizing the link since $\lambda_{l} \ll C_{l}$ also results in a loss-free operation. Therefore, we need $\lambda_{l} \approx C_{l}$ where possible. This motivates our adaptive algorithm which is given by the following.

Algorithm II.1: Each link $l \in \mathcal{L}$ updates its virtual capacity according to the ordinary differential equation (ODE)

$$
\dot{\tilde{C}}_{l}=-\alpha \frac{1}{\partial p_{l}\left(\lambda_{l}, \tilde{C}_{l}\right) / \partial \tilde{C}_{l}}\left(C_{l}-\lambda_{l}\right)
$$

where $\alpha>0$ is the damping factor. To avoid $\tilde{C}_{l}$ becoming negative, we set $\dot{\widetilde{C}}_{l}=0$ whenever $\tilde{C}_{l}=0$ and $\lambda_{l}>C_{l}$.

Note that the adaptive algorithm increases the virtual capacity whenever the total flow into the link is lower than the capacity of the link. This results in a reduced number of marks to the users using that link which results in the users increasing their rates. Also, note that the algorithm is distributed in nature and does not require any transfer of information from other nodes/links. This algorithm is a modification of the one presented in [6]. Here, we have introduced the derivative of the marking rate with respect to the virtual capacity in the adaptive algorithm. This modification is necessary to our proof of convergence. However, simulation studies in [6] suggest that the algorithm may converge without the derivative term and the proof of this is an open problem.

The complete system of differential equations governing the behavior of the network can now be written down as

$$
\begin{aligned}
\frac{d x_{r}(t)}{d t} & =\left(\Delta_{r}-\frac{\beta}{U_{r}^{\prime}\left(x_{r}(t)\right)} \sum_{l: l \in r} p_{l}\left(\lambda_{l}, \tilde{C}_{l}\right)\right) \quad \forall r \in \mathcal{R} \\
\frac{d \tilde{C}_{l}}{d t} & =-\frac{\alpha}{\partial p_{l}\left(\lambda_{l}, \tilde{C}_{l}\right) / \partial \tilde{C}_{l}}\left(C_{l}-\lambda_{l}\right) \quad \forall l \in \mathcal{L} .
\end{aligned}
$$

\section{Stability Analysis of the Reduced System}

We consider the system of differential equations governing the behavior of the network

$$
\begin{aligned}
\frac{d x_{r}(t)}{d t} & =\left(\Delta_{r}-\frac{\beta}{U_{r}^{\prime}\left(x_{r}(t)\right)} \sum_{l: l \in r} p_{l}\left(\lambda_{l}, \tilde{C}_{l}\right)\right) \quad \forall r \in \mathcal{R} \\
\frac{d \tilde{C}_{l}}{d t} & =-\frac{\alpha}{\partial p_{l}\left(\lambda_{l}, \tilde{C}_{l}\right) / \partial \tilde{C}_{l}}\left(C_{l}-\lambda_{l}\right) \quad \forall l \in \mathcal{L} .
\end{aligned}
$$

By letting $\tau=\alpha t$, we can rewrite (11)-(12) as

$$
\begin{aligned}
\alpha \frac{d x_{r}(t)}{d \tau} & =\left(\Delta_{r}-\frac{\beta}{U_{r}^{\prime}\left(x_{r}(\tau)\right)} \sum_{l: l \in r} p_{l}\left(\lambda_{l}, \tilde{C}_{l}\right)\right) \forall r \in \mathcal{R} \\
\frac{d \tilde{C}_{l}}{d \tau} & =-\frac{1}{\partial p_{l}\left(\lambda_{l}, \tilde{C}_{l}\right) / \partial \tilde{C}_{l}}\left(C_{l}-\lambda_{l}\right) \quad \forall l \in \mathcal{L} .
\end{aligned}
$$

This reduces (9)-(10) to (13)-(14), which is a standard singular perturbation model of the system [10]. If we set $\alpha=0$, we get

$$
\left(\Delta_{r}-\frac{\beta}{U_{r}^{\prime}\left(x_{r}(\tau)\right)} \sum_{l: l \in r} p_{l}\left(\lambda_{l}, \tilde{C}_{l}\right)\right)=0 .
$$


We know from [6] that there exists a unique root $\left\{x_{r}^{*}\right\}$ to the above algebraic equation that also solves the optimization problem given by (7). Let $\lambda_{l}^{*}=\sum_{r: l \in r} x_{r}^{*}$. The reduced-system is given by

$x^{*}=\arg \max _{x} \sum_{r} \Delta_{r} U_{r}\left(x_{r}\right)-\beta \sum_{l \in \mathcal{L}} \int_{0}^{\sum_{r: l \in r} x_{r}} p_{l}\left(z, \tilde{C}_{l}\right) d z$,

$\dot{\widetilde{C}}_{l}=-\frac{1}{\partial p_{l}\left(\lambda_{l}^{*}, \tilde{C}_{l}\right) / \partial \tilde{C}_{l}}\left(C_{l}-\lambda_{l}^{*}\right) \quad \forall l \in \mathcal{L}$.

This model assumes that $x=\left(x_{r}, r \in \mathcal{R}\right)$, whose velocity given by $\dot{x}$ is large when $\alpha$ is small, converges rapidly to the equilibrium point of (13). The boundary-layer system is given by

$$
\frac{d x_{r}(t)}{d t}=\left(\Delta_{r}-\frac{\beta}{U_{r}^{\prime}\left(x_{r}(t)\right)} \sum_{l: l \in r} p_{l}\left(\lambda_{l}, \tilde{C}_{l}\right)\right) \quad \forall r \in \mathcal{R}
$$

where $\left\{\tilde{C}_{l}\right\}$ is treated as a constant parameter. It is shown in [4] that the boundary-layer system is globally asymptotically stable. We will study the stability of the reduced system in this section. We will first define an invariant point of the algorithm given in (17) as follows.

Definition III.1: A point $\left\{\hat{C}_{l}\right\}$ is an invariant point if

$$
\begin{aligned}
& \dot{\hat{C}}_{l}=0 \quad \forall\left\{l: \hat{C}_{l}<\infty\right\} \\
& \dot{\hat{C}}_{l} \geq 0 \quad \forall\left\{l: \hat{C}_{l}=\infty\right\} .
\end{aligned}
$$

Before discussing the stability of the adaptive algorithm, we will first prove the existence of virtual capacities at each link so that socially optimal operation can be guaranteed and that these virtual capacities are also an invariant point of the system. This shows that there exists at least one invariant point of the reduced-system that guarantees a socially optimal operation. We will then proceed to show that all the invariant points of the reduced-system in (16)-(17) ensure socially optimal operation (i.e., they result in system optimal rates). We then prove the global asymptotic stability of the reduced-system.

- Let $\left\{\bar{x}_{r}\right\}$ be the system optimal rates obtained by solving $\operatorname{SYSTEM}(U, S, C)$ and let $\bar{\lambda}_{l}=\sum_{r: l \in r} \bar{x}_{r}$ for all $l \in \mathcal{L}$.

- Let $x^{*}\left(\left\{\hat{C}_{l}\right\}\right)=\left(x_{r}^{*}\left(\left\{\hat{C}_{l}\right\}\right), r \in \mathcal{R}\right)$ be the solution of (16) with $\tilde{C}_{l}=\hat{C}_{l}$ for all $l \in \mathcal{L}$, where $\left\{\hat{C}_{l}\right\}$ is an invariant point defined in Definition III.1. Let $\lambda_{l}^{*}\left(\left\{\hat{C}_{l}\right\}\right)=\sum_{r_{i} l \in r} x_{r}^{*}\left(\left\{\hat{C}_{l}\right\}\right)$ for all $l \in \mathcal{L}$ and $\lambda^{*}\left(\left\{\hat{C}_{l}\right\}\right)=\left(\lambda_{l}^{*}\left(\left\{\hat{C}_{l}\right\}\right), l \in \mathcal{L}\right)$.

- $\mathcal{L}_{a}\left(\left\{\hat{C}_{l}\right\}\right)=\left\{\right.$ set of congested links i.e., $\lambda_{l}^{*}\left(\left\{\hat{C}_{l}\right\}\right)=$ $C_{l}$, at an invariant point $\left\{\hat{C}_{l}\right\}$ of the algorithm $\}$.

- $\mathcal{L}_{o}=\left\{\right.$ set of congested links i.e., $\bar{\lambda}_{l}=C_{l}$, at the solution of $\operatorname{SYSTEM}(U, S, C)\}$.

\section{A. Existence}

We show in this section that there exists virtual capacities at each link that allows the congestion controllers obtained from the penalty function formulation to drive the system toward the solution of SYSTEM $(U, S, C)$. Before showing this result, we will state and prove some lemmas which will be used in the existence proof.

Consider the single-link optimization problem

$$
\begin{gathered}
\max _{\left\{x_{r}\right\}: l \in r} \sum_{r: l \in r} \Delta_{r} U_{r}\left(x_{r}\right) \\
\text { subject to } \sum_{\substack{r: l \in r \\
x_{r}}} x_{r} \leq 0 \quad \forall r: l \in C_{l} \\
\quad \forall r
\end{gathered}
$$

The Lagrangian of this maximization problem can be written as

$$
\mathcal{G}\left(x, \nu_{l}\right)=\sum_{r: l \in r} \Delta_{r} U_{r}\left(x_{r}\right)-\nu_{l}\left(\sum_{r: l \in r} x_{r}-C_{l}\right)
$$

where $\nu_{l}$ is the Lagrange multiplier. Therefore, the optimal solution satisfies [9]

$$
\begin{aligned}
& \nu_{l}=0 \quad \text { if } \sum_{r: l \in r} x_{l}<C_{l} \\
& \nu_{l}>0 \quad \text { if } \sum_{r: l \in r} x_{l}=C_{l} .
\end{aligned}
$$

Lemma III.1: The feedback from the link in a single link case when $\tilde{C}_{l}=\tilde{C}_{l}^{(d)}$, where $\tilde{C}_{l}^{(d)}$ is defined in Assumption 1 , is equal to the Lagrange multiplier obtained by solving the constrained optimization problem in (19). In other words

$$
\nu_{l}=\beta p_{l}\left(C_{l}, \tilde{C}_{l}^{(d)}\right)
$$

Proof: See Appendix VII.A

Next, let us consider the Lagrangian of $\operatorname{SYSTEM}(U, S, C)$

$$
\mathcal{H}\left(\lambda_{l}, \mu_{l}\right)=\max _{\left\{x_{r}\right\}} \sum_{r} \Delta_{r} U_{r}\left(x_{r}\right)-\sum_{l \in \mathcal{L}} \mu_{l}\left(\sum_{r: j \in r} x_{j}-C_{j}\right)
$$

where $\mu=\left(\mu_{l}: l \in \mathcal{L}\right)$ is the Lagrange multiplier. Therefore, we know that the optimal solution satisfies [9]

$$
\begin{aligned}
& \mu_{l}=0 \text { if } \sum_{r: l \in r} x_{l}<C_{l} \\
& \mu_{l}>0 \text { if } \sum_{r: l \in r} x_{l}=C_{l}
\end{aligned}
$$

Let $\bar{x}_{r}$ denote the system optimal rates obtained by solving $\operatorname{SYSTEM}(U, S, C)$ and let $\bar{\lambda}_{l}=\sum_{r: l \in r} \bar{x}_{r}$. By writing the first-order necessary conditions, we get

$$
\bar{\lambda}_{l}=\sum_{r: l \in r} U_{r}^{\prime-1}\left[\sum_{j: j \in r} \frac{\mu_{j}}{\Delta_{r}}\right] \text {. }
$$

Lemma III.2: $\mu_{l} \leq \nu_{l}$ for all $l \in \mathcal{L}$.

Proof: See Appendix VII.B.

Theorem III.1: (Existence Theorem) There exists virtual capacities $\left\{\tilde{C}_{l}\right\}$ at each link $l \in \mathcal{L}$ such that the unique maximum of the optimization problem given by (7) also solves $\operatorname{SYSTEM}(U, S, C)$.

Proof: The optimization problem is given by

$$
\max _{\left\{x_{r}\right\}} \sum_{r} \Delta_{r} U_{r}\left(x_{r}\right)-\beta \sum_{l \in \mathcal{L}} \int_{0}^{\lambda_{l}} p_{l}\left(z, \tilde{C}_{l}\right) d z .
$$


Let $x^{*}$ be the solution to the above optimization problem and let $\lambda_{l}^{*}=\sum_{r: l \in r} x_{r}^{*}$ for all $l \in \mathcal{L}$. Writing down the first-order necessary conditions, we get

$$
x_{r}^{*}=U_{r}^{\prime-1}\left(\frac{\beta}{\Delta_{r}} \sum_{k: k \in r} p_{k}\left(\lambda_{k}^{*}, \tilde{C}_{k}\right) .\right) .
$$

Therefore, for each link $l \in \mathcal{L}$

$$
\lambda_{l}^{*}=\sum_{r: l \in r} U_{r}^{\prime-1}\left(\frac{\beta}{\Delta_{r}} \sum_{k: k \in r} p_{k}\left(\lambda_{k}^{*}, \tilde{C}_{k}\right) .\right) .
$$

Now, we need to show that there exists $\tilde{C}_{l}, l \in \mathcal{L}$ that solves

$$
\bar{\lambda}_{l}=\sum_{r: l \in r} U_{r}^{\prime-1}\left[\frac{\beta}{\Delta_{r}} \sum_{k: k: \in r} p_{k}\left(\bar{\lambda}_{k}, \tilde{C}_{k}\right)\right] .
$$

Comparing (26) and (25), let us set

$$
\mu_{l}=\beta p_{l}\left(\bar{\lambda}_{l}, \tilde{C}_{l}\right) \quad \forall l \in \mathcal{L} .
$$

If $\bar{\lambda}_{l}<C_{l}$, then from (24) $\mu_{l}=\beta p_{l}\left(\bar{\lambda}_{l}, \tilde{C}_{l}\right)=0$. Therefore, set $\tilde{C}_{l}=\infty$ for such links. We now need to show that there exists a $\tilde{C}_{l}$ which will satisfy the above equation for all links for which $\bar{\lambda}_{l}=C_{l}$. From Lemma (III.1), there exists a $\tilde{C}_{l}^{(d)}$ that solves $\nu_{l}=\beta p_{l}\left(C_{l}, \tilde{C}_{l}\right)$. Also, from Lemma (III.2), $\mu_{l} \leq \nu_{l}$. Therefore, there exists a $\tilde{C}_{l} \geq \tilde{C}_{l}^{(d)}$ for all $l \in \mathcal{L}$ such that

$$
\mu_{l}=\beta p_{l}\left(\bar{\lambda}_{l}, \tilde{C}_{l}\right) \quad \forall l \in\left\{j \in \mathcal{L}: \bar{\lambda}_{j}<C_{j}\right\} .
$$

This shows the existence of a $\widetilde{C}_{l}$ at each link $l \in \mathcal{L}$ such that the unique maximum of the optimization problem given by (7) also solves the convex program given by $\operatorname{SYSTEM}(U, S, C)$.

\section{B. Properties of Invariant Points}

In this section, we will show that the invariant points of the reduced-system given by (16)-(17) indeed lead to the socially optimal operation. Moreover, the rates obtained at the invariant points are the system optimal rates.

Lemma III.3: The user rates $\left(\lambda^{*}\left(\left\{\hat{C}_{l}\right\}\right)\right)$ at an invariant point $\left\{\hat{C}_{l}\right\}$ satisfies the following conditions:

$$
\begin{array}{ll}
\lambda_{l}^{*}=C_{l} & \forall l \in\left\{j \in \mathcal{L}: \hat{C}_{j}<\infty\right\} \\
\lambda_{l}^{*}<C_{l} & \forall l \in\left\{j \in \mathcal{L}: \hat{C}_{j}=\infty\right\} .
\end{array}
$$
point iff

Proof: From (17) and Definition III.1, $\hat{C}_{l}$ is an invariant

1) $\lambda_{l}^{*}=C_{l}$ if $\hat{C}_{l}<\infty$;

2) $\lambda_{l}^{*}<C_{l}$ and $\hat{C}_{l}=\infty$;

3) $\lambda_{l}^{*}>C_{l}$ and $\hat{C}_{l}=0$.

Therefore, we need to show that condition 3 ) is not possible. Assume that it is possible, i.e., $\hat{C}_{l}=0$ and $\lambda_{l}^{*}>C_{l}$. Also, from Assumption 1 , we know that $\tilde{C}_{l}^{(d)} \geq 0$. From Assumption 1 and the fact that $U_{r}^{\prime-1}$ is a strictly decreasing function, we know that

$$
\begin{aligned}
C_{l} & =\sum_{r: l \in r} U_{r}^{\prime-1}\left[\frac{\beta}{\Delta_{r}} p_{l}\left(C_{l}, \tilde{C}_{l}^{(d)}\right)\right] \\
& \geq \sum_{r: l \in r} U_{r}^{\prime-1}\left[\frac{\beta}{\Delta_{r}} p_{l}\left(C_{l}, 0\right)\right] .
\end{aligned}
$$

We also know that

$$
\begin{aligned}
\lambda_{l}^{*} & =\sum_{r: l \in r} U_{r}^{\prime-1}\left[\frac{\beta}{\Delta_{r}} \sum_{j: j \in r} p_{j}\left(\lambda_{j}^{*}, \hat{C}_{j}\right)\right] \\
& =\sum_{r: l \in r} U_{r}^{\prime-1}\left[\frac{\beta}{\Delta_{r}} p_{l}\left(\lambda_{l}^{*}, 0\right)+\frac{\beta}{\Delta_{r}} \sum_{j: j \in r, j \neq l} p_{j}\left(\lambda_{j}^{*}, \hat{C}_{j}\right)\right] .
\end{aligned}
$$

However, $p_{l}(q, s)$ is increasing in $q$. Therefore

$$
\begin{aligned}
& \beta p_{l}\left(\lambda_{l}^{*}, 0\right)>\beta p_{l}\left(C_{l}, 0\right) \\
& \Rightarrow \beta p_{l}\left(\lambda_{l}^{*}, 0\right)+\beta \sum_{j: j \in r, j \neq l} p_{j}\left(\lambda_{j}^{*}, \hat{C}_{j}\right)>\beta p_{l}\left(C_{l}, 0\right) .
\end{aligned}
$$

Since, $U_{r}^{\prime-1}$ is a strictly decreasing function and $\Delta_{r}>0$, we have

$$
\begin{aligned}
C_{l} & <\sum_{r: l \in r} U_{r}^{\prime-1}\left[\frac{\beta}{\Delta_{r}} p_{l}\left(\lambda_{l}^{*}, 0\right)+\sum_{j: j \in r, j \neq l} \frac{\beta}{\Delta_{r}} p_{j}\left(\lambda_{j}^{*}, \hat{C}_{j}\right)\right] \\
& <\sum_{r: l \in r} U_{r}^{\prime-1}\left[\frac{\beta}{\Delta_{r}} p_{l}\left(C_{l}, 0\right)\right] \leq C_{l}
\end{aligned}
$$

which is a contradiction. Hence, condition 3) is not possible. Therefore, either conditions 1) or 2) should be satisfied when $\dot{\tilde{C}}_{l}=0$.

Theorem III.2: The user rates obtained at any invariant point of the system given in (16)-(17) equals the system optimal rates.

Proof: Suppose there exists an invariant point $\left\{\hat{C}_{l}\right\}$, such that $\lambda^{*}\left(\left\{\hat{C}_{l}\right\}\right)$ does not solve $\operatorname{SYSTEM}(U, S, C)$. Let $\mathcal{J}(x)$ denote the objective function in the maximization problem given by (7). Then, the maximum value of the optimization-problem (7) is

$$
\mathcal{J}\left(x^{*}\right)=\left\{\begin{array}{l}
\sum_{r} \Delta_{r} U_{r}\left(x_{r}^{*}\right)-\beta \sum_{l \in \mathcal{L}_{a}} \int_{0}^{C_{l}} p_{l}\left(z, \hat{C}_{l}\right) d z \\
-\beta \sum_{l \in \mathcal{L} \backslash \mathcal{L}_{a}} \int_{0}^{\lambda_{l}^{*}} p_{l}\left(z, \hat{C}_{l}\right) d z
\end{array} .\right.
$$

Equation (27) can be rewritten as

$$
\mathcal{J}\left(x^{*}\right)=\left\{\begin{array}{l}
\sum_{r} \Delta_{r} U_{r}\left(x_{r}^{*}\right)-\beta \sum_{l \in \mathcal{L}_{a} \cap \mathcal{L}_{o}} \int_{0}^{C_{l}} p_{l}\left(z, \hat{C}_{l}\right) d z \\
-\beta \sum_{l \in \mathcal{L}_{a} \cap\left(\mathcal{L} \backslash \mathcal{L}_{o}\right)} \int_{0}^{C_{l}} p_{l}\left(z, \hat{C}_{l}\right) d z \\
-\beta \sum_{l \in \mathcal{L} \backslash \mathcal{L}_{a}} \int_{0}^{\lambda_{l}^{*}} p_{l}\left(z, \hat{C}_{l}\right) d z
\end{array} .\right.
$$

Also

$$
\mathcal{J}(\bar{x})=\left\{\begin{array}{l}
\sum_{r} \Delta_{r} U_{r}\left(\bar{x}_{r}\right)-\beta \sum_{l \in \mathcal{L}_{a} \cap \mathcal{L}_{o}} \int_{0}^{C_{l}} p_{l}\left(z, \hat{C}_{l}\right) d z \\
-\beta \sum_{l \in \mathcal{L}_{a} \cap\left(\mathcal{L} \backslash \mathcal{L}_{o}\right)} \int_{0}^{\bar{\lambda}_{l}} p_{l}\left(z, \hat{C}_{l}\right) d z- \\
\beta \sum_{l \in \mathcal{L} \backslash \mathcal{L}_{a}} \int_{0}^{\bar{\lambda}_{l}} p_{l}\left(z, \hat{C}_{l}\right) d z
\end{array} .\right.
$$

However, we know that

1) $\sum_{r} \Delta_{r} U_{r}\left(\bar{x}_{r}\right) \geq \sum_{r} \Delta_{r} U_{r}\left(x_{r}^{*}\right)$, as $\left\{\bar{x}_{r}\right\}$ is the unique maximum point of $\operatorname{SYSTEM}(U, S, C)$; 
2)

$$
\begin{aligned}
-\beta \sum_{l \in \mathcal{L}_{a} \cap\left(\mathcal{L} \backslash \mathcal{L}_{o}\right)} \int_{0}^{\bar{\lambda}_{l}} p_{l}\left(z, \hat{C}_{l}\right) d z & \\
& \geq-\beta \sum_{l \in \mathcal{L}_{a} \cap\left(\mathcal{L} \backslash \mathcal{L}_{o}\right)} \int_{0}^{C_{l}} p\left(z, \hat{C}_{l}\right) d z,
\end{aligned}
$$

as $\bar{\lambda}_{l}<C_{l}$ for all $l \in \mathcal{L}_{a} \cap\left(\mathcal{L} \backslash \mathcal{L}_{o}\right)$;

3) $\begin{aligned} & \beta \sum_{l \in \mathcal{L} \backslash \mathcal{L}_{a}} \int_{0}^{\bar{\lambda}_{l}} p_{l}\left(z, \hat{C}_{l}\right) d z=0 \text { as } \hat{C}_{l}=\infty \text { for all } l \in \\ & \mathcal{L} \backslash \mathcal{L}_{a} .\end{aligned}$ Therefore

$$
\mathcal{J}(\bar{x})>\mathcal{J}\left(x^{*}\right)
$$

However, the invariant point is the optimal solution to the optimization problem (7) which leads to a contradiction. Therefore, the rates obtained at the invariant point of the algorithm also solve the system problem $\operatorname{SYSTEM}(U, S, C)$.

\section{Stability of the Reduced-System}

In this section, we will prove that the reduced-system given by (16)-(17) is globally asymptotically stable using a Lyapunov function. We will make the following assumption to show the stability of the reduced-system.

Assumption 2: All the links in the network are fully utilized i.e., the solution to $\operatorname{SYSTEM}(U, S, C)$ satisfies, $\bar{\lambda}_{l}=C_{l}$ for all $l \in \mathcal{L}$.

Let us consider the function

$$
\mathcal{W}\left(\lambda^{*}(\tilde{C})\right)=\sum_{l \in \mathcal{L}}\left(C_{l}-\lambda_{l}^{*}\right)^{2}
$$

$\mathcal{W}\left(\lambda^{*}\right)$ is a positive convex function. Let $M$ be the number of links in the network, i.e., $M=\operatorname{card}(\mathcal{L})$. and $N$ be the number of routes. We will show that $\mathcal{W}\left(\lambda_{l}^{*}\right)$ is a Lyapunov function for the system described by (16)-(17). Let us now consider the time derivative of $\mathcal{W}\left(\lambda^{*}\right)$. We get

$$
\begin{aligned}
\frac{d \mathcal{W}\left(\lambda^{*}\right)}{d t}= & \sum_{l \in \mathcal{L}} \frac{\partial W\left(\lambda_{l}^{*}\right)}{\partial \lambda_{l}^{*}} \frac{d \lambda_{l}^{*}}{d t} \\
= & \sum_{l \in \mathcal{L}}-2 .\left(C_{l}-\lambda_{l}^{*}\right) \sum_{k \in \mathcal{L}} \frac{\partial \lambda_{l}^{*}}{\partial \tilde{C}_{k}} \frac{d \tilde{C}_{k}}{d t} \\
= & -2 \sum_{l \in \mathcal{L}}\left(C_{l}-\lambda_{l}^{*}\right) \\
& \times \sum_{k \in \mathcal{L}} \frac{d \lambda_{l}^{*}}{d \tilde{C}_{k}} \frac{-\left(C_{k}-\lambda_{k}^{*}\right)}{\partial p_{k}\left(\lambda_{k}^{*}, \tilde{C}_{k}\right) / \partial \tilde{C}_{k}} \\
= & -2 \phi^{T} \Lambda B^{-1} \phi
\end{aligned}
$$

where

$$
\Lambda:=\left[\begin{array}{ccc}
\frac{d \lambda_{1}^{*}}{d \tilde{C}_{1}} & \cdots & \frac{d \lambda_{1}^{*}}{d \tilde{C}_{M}} \\
\frac{d \lambda_{2}^{2}}{d \tilde{C}_{1}} & \cdots & \frac{d \lambda_{2}^{2}}{d \tilde{C}_{M}} \\
\vdots & & \vdots \\
\frac{d \lambda_{M}^{*}}{d \tilde{C}_{1}} & \cdots & \frac{d \lambda_{M}^{*}}{d \tilde{C}_{M}}
\end{array}\right], \phi:=\left[\begin{array}{c}
C_{1}-\lambda_{1}^{*} \\
C_{2}-\lambda_{2}^{*} \\
\vdots \\
C_{M}-\lambda_{M}^{*}
\end{array}\right]
$$

$$
B:=\left[\begin{array}{cccc}
-\frac{\partial p_{1}\left(\lambda_{1}^{*}, \tilde{C}_{1}\right)}{\partial \tilde{C}_{1}} & 0 & \cdots & 0 \\
0 & -\frac{\partial p_{2}\left(\lambda_{2}^{*}, \tilde{C}_{2}\right)}{\partial \tilde{C}_{2}} & \cdots & 0 \\
\vdots & \vdots & \cdots & 0 \\
0 & 0 & \cdots & -\frac{\partial p_{M}\left(\lambda_{M}^{*}, \tilde{C}_{M}\right)}{\partial \widetilde{C}_{M}}
\end{array}\right]
$$

We know that $B>0$ from Assumption 1. We also know that for all $r \in \mathcal{R}$

$$
x_{r}^{*}=U_{r}^{\prime-1}\left(\frac{\beta}{\Delta_{r}} \sum_{k: k \in r} p_{k}\left(\lambda_{k}^{*}, \tilde{C}_{k}\right)\right) .
$$

Therefore, differentiating the above expression with respect to $\tilde{C}_{i}$, we get

$$
\frac{d x_{r}^{*}}{d \tilde{C}_{i}}=\left\{\begin{array}{l}
\frac{1}{U_{r}^{\prime \prime}\left(x_{r}^{*}\right)}\left(\frac{\beta}{\Delta_{r}} \sum_{k: k \in r} \frac{\partial p_{k}\left(\lambda_{k}^{*}, \tilde{C}_{k}\right)}{\partial \lambda_{k}^{*}} \sum_{j: k \in j} \frac{d x_{j}}{d \tilde{C}_{i}}\right) \\
+\frac{1}{U_{r}^{\prime \prime}\left(x_{r}^{*}\right)} \frac{\beta}{\Delta_{r}} \frac{\partial p_{i}\left(\lambda_{i}^{*}, \tilde{C}_{i}\right)}{\partial \tilde{C}_{i}} I_{i \in r} \forall r \in \mathcal{R}, \forall i \in \mathcal{L}
\end{array} .\right.
$$

where $I_{z}$ is the indicator function that event $z$ occurs. Define the matrix $A$ as

$$
A_{r q}= \begin{cases}-\beta \sum_{\substack{k: k \in r \\ k \in q}} \frac{\partial p_{k}\left(\lambda_{k}^{*}, \tilde{C}_{k}\right)}{\partial \lambda_{k}} & r \neq q \\ \Delta_{r} U_{r}^{\prime \prime}\left(x_{r}^{*}\right)-\beta \sum_{k: k \in r} \frac{\partial p_{k}\left(\lambda_{k}^{*}, \tilde{C}_{k}\right)}{\partial \lambda_{k}} & r=q\end{cases}
$$

We can now rewrite (34) as

$$
A .\left[\begin{array}{c}
\frac{d x_{1}^{*}}{d \tilde{C}_{i}} \\
\frac{d x_{2}^{*}}{d \tilde{C}_{i}} \\
\vdots \\
\frac{d x_{N}^{*}}{d \tilde{C}_{i}}
\end{array}\right]=\left[\begin{array}{c}
\beta \frac{\partial p_{i}\left(\lambda_{i}^{*}, \tilde{C}_{i}\right)}{\partial \tilde{C}_{i}} I_{i \in 1} \\
\beta \frac{\partial p_{i}\left(\lambda_{i}^{*}, \tilde{C}_{i}\right)}{\partial \tilde{C}_{i}} I_{i \in 2} \\
\vdots \\
\beta \frac{\partial p_{i}\left(\lambda_{i}^{*} \tilde{C}_{i}\right)}{\partial \tilde{C}_{i}} I_{i \in N}
\end{array}\right] \quad \forall i \in \mathcal{L}
$$

However, $A$ is the Hessian of

$$
\max _{\left\{x_{r}\right\}} \sum_{r \in \mathcal{R}} \Delta_{r} U_{r}\left(x_{r}\right)-\beta \sum_{l \in \mathcal{L}} \int_{0}^{\lambda_{l}^{*}} p_{l}\left(z, \tilde{C}_{l}\right) d z .
$$

Since, (37) is a strictly concave function, $A$ is strictly negative-definite, i.e., $A<0$, for all $\left\{\tilde{C}_{l}\right\}$. Therefore, $A^{-1}<0$. Let

$$
\Omega:=\left[\begin{array}{ccc}
\frac{d x_{1}^{*}}{d \tilde{C}_{1}} & \cdots & \frac{d x_{1}^{*}}{d \tilde{C}_{M}} \\
\frac{d x_{2}^{*}}{d \tilde{C}_{1}} & \cdots & \frac{d x_{2}^{*}}{d \tilde{C}_{M}} \\
\vdots & & \vdots \\
\frac{d x_{N}^{*}}{d \tilde{C}_{1}} & \cdots & \frac{d x_{N}^{*}}{d \tilde{C}_{M}}
\end{array}\right]_{N \times M}
$$

and

$$
D:=\beta\left[\begin{array}{ccc}
\frac{\partial p_{1}\left(\lambda_{1}^{*}, \tilde{C}_{1}\right)}{\partial \tilde{C}_{1}} I_{1 \in 1} & \cdots & \frac{\partial p_{M}\left(\lambda_{M}^{*}, \tilde{C}_{M}\right)}{\partial \tilde{C}_{M}} I_{M \in 1} \\
\frac{\partial p_{1}\left(\lambda_{1}^{*}, \tilde{C}_{1}\right)}{\partial \tilde{C}_{1}} I_{1 \in 2} & \cdots & \frac{\partial p_{M}\left(\lambda_{M}^{*}, \tilde{C}_{M}\right)}{\partial \tilde{C}_{M}} I_{M \in 2} \\
\vdots & & \vdots \\
\frac{\partial p_{1}\left(\lambda_{1}^{*}, \dot{\tilde{C}}_{1}\right)}{\partial \tilde{C}_{1}} I_{1 \in N} & \cdots & \frac{\partial p_{M}\left(\lambda_{M}^{*}, \hat{C}_{M}\right)}{\partial \tilde{C}_{M}} I_{M \in N}
\end{array}\right]=-\beta S B
$$


where $S^{T}$ is the routing matrix defined in Section II. Now, we can rewrite (36) as

$$
\begin{aligned}
& A \Omega=D \\
& \Rightarrow \Omega=A^{-1} D \\
& \Rightarrow S^{T} \Omega=S^{T} A^{-1} D \\
& \Rightarrow \Lambda=-\beta S^{T} A^{-1} S B .
\end{aligned}
$$

The next theorem shows that the system described by (16)-(17) is globally asymptotically stable and the invariant points solve $\operatorname{SYSTEM}(U, S, C)$.

Theorem III.3: The strictly convex function $\mathcal{W}\left(\lambda^{*}\right)$ is a Lyapunov function for the system of differential equations (16)-(17). The unique value $\left\{\bar{\lambda}_{l}\right\}$ at $\mathcal{W}\left(\lambda^{*}\right)=0$ is a stable point of the system, to which all trajectories converge. Moreover, $\left\{\bar{\lambda}_{l}\right\}$ solves SYSTEM $(U, S, C)$.

Proof: We know that $\mathcal{W}\left(\lambda^{*}\right)$ is a strictly convex function and it has a unique minimizing value. Further, from (31)

$$
\frac{d \mathcal{W}\left(\lambda^{*}\right)}{d t}=-2 \phi^{T} \Lambda B^{-1} \phi .
$$

Using (41), we can rewrite (42) as

$$
\frac{d \mathcal{W}\left(\lambda^{*}\right)}{d t}=2 \beta \phi^{T} S^{T} A^{-1} S B B^{-1} \phi=2 \beta \phi^{T} S^{T} A^{-1} S \phi .
$$

Since $A^{-1}<0$, we get $S A^{-1} S^{T} \leq 0$. Hence

$$
\frac{d \mathcal{W}\left(\lambda^{*}\right)}{d t} \leq 0 \quad \forall t
$$

Since, we have assumed that the original problem $\operatorname{SYSTEM}(U, S, C)$ has all links utilized i.e., $\bar{\lambda}_{l}=C_{l}$, from Lemma III.3, we know that all the invariant points of the system has $\lambda_{l}^{*}=C_{l}$. Therefore, $\dot{\mathcal{W}}\left(\lambda^{*}(\tilde{C})\right)=0$ at the invariant points. Therefore, from LaSalle's invariance principle [10], the system of differential functions (16)-(17) converge to the invariant points of the algorithm.

Thus, we have shown that the reduced-system is asymptotically stable with the invariant points leading to the system optimal rates when all the links are fully utilized in the system optimal solution, i.e., $\bar{\lambda}_{l}=C_{l}$, for all links $l \in \mathcal{L}$. Note that even though the $\lambda_{l}^{*}=C_{l}$, for all $l \in \mathcal{L}$ is the unique equilibrium point, there might be many values of $\tilde{C}$ that achieves this equilibrium point. For this reason, we use LaSalle's invariance principle in the proof to show stability. We will now show that the entire system is semiglobally exponentially stable under certain conditions. Toward this, we will first show that both the reduced-system and the boundary-layer system are semiglobally exponentially stable.

\section{Stability of The Overall System}

In this section, we will show that the singularly perturbed system is semiglobally exponentially stable under an additional assumption. We will assume that the system satisfies the following assumption.

Assumption 3: Each link has at least one flow that passes only through it.
We will make use of Assumption 3 to show that there exists an $\alpha$ small enough such that the entire system given by (11)-(12) or equivalently (13)-(14) is semiglobally exponentially stable. Note that Assumption 3 encompasses Assumption 2 and also ensures that there exists an unique $\tilde{C}$ that achieves the equilibrium point.

We define the vectors, $\lambda^{*}:=\left(\lambda_{l}^{*}, l \in \mathcal{L}\right), \tilde{C}:=\left(\tilde{C}_{l}, l \in\right.$ $\mathcal{L}), x:=\left(x_{r}, r \in \mathcal{R}\right)$, and $C-\lambda^{*}:=\left(C_{l}-\lambda_{l}^{*}, l \in \mathcal{L}\right)$. The original system (13)-(14) can be written as

$$
\begin{aligned}
\alpha \dot{x} & =f(x, \tilde{C}) \\
\tilde{\tilde{C}} & =g(x, \tilde{C})
\end{aligned}
$$

where $f$ is a continuously differentiable function in $x$ and $\tilde{C}$, and

$$
g_{l}(x, \tilde{C})=\left\{\begin{array}{cc}
C-\sum_{r: r \in l} x_{r} & \text { if } \tilde{C}_{l}>0 \\
\left(C-\sum_{r: r \in l} x_{r}\right)^{+} & \text {if } \tilde{C}_{l}=0
\end{array}\right.
$$

Note that $g(x, \tilde{C})$ is continuously differentiable everywhere except at those points where $\tilde{C}_{i}=0$ and $\lambda_{i}>C_{i}$ for some $i$. We will now state a sequence of lemmas and theorems that would be useful to prove the main result of this section.

Lemma IV.1: Let $x^{*}(\tilde{C})$ be the unique root of the equation $f(x, \widetilde{C})=0$. Then, $x^{*}(\widetilde{C})$ is a continuously differentiable function of $\tilde{C}$.

Proof: See Appendix VII.C.

The following lemma gives an upper bound to the virtual capacity at a link in the reduced system (17). This lemma is useful in showing that the virtual capacity in the reduced system belongs to a compact set. We can then use this to bound the virtual capacity in the original singular perturbation system (11)-(12).

Lemma IV.2: Let $\hat{C}_{l}$ solve

$$
\beta p_{l}\left(C_{l}, \hat{C}_{l}\right)=\Delta_{j} U_{j}^{\prime}\left(C_{l}\right)
$$

where $U_{j}($.$) is the utility function of one of the flows that passes$ only through link $l$. Then, there exists a $\hat{t}<\infty$, such that for all $t \geq \hat{t}, \tilde{C}_{l}(t)$ in the reduced system belongs to $\left[0, n_{l} \hat{C}_{l}\right]$, where $n_{l}>1$.

\section{Proof: See Appendix VII.D}

Due to the above lemma, we will assume that $\tilde{C}_{l}(0) \in$ $\left[0, n_{l} \hat{C}_{l}\right]$, where $n_{l}$ is some arbitrary number greater than one and therefore $\tilde{C}_{l}(t) \in\left[0, n_{l} \hat{C}_{l}\right]$ for all $t \geq 0$. Define

$$
\begin{array}{r}
\mathcal{B}_{\tilde{C}}=\left\{\left(u_{1}, u_{2}, \ldots, u_{M}\right): 0 \leq u_{i} \leq n_{i} \hat{C}_{i}, \quad n_{i}>1,\right. \\
\forall 1 \leq i \leq M,\}
\end{array}
$$

where $M$ is the number of links in the network, i.e., $M=$ $\operatorname{card}(\mathcal{L})$. We know that, $\tilde{C}(t) \in \mathcal{B}_{\tilde{C}}$, for all $t \geq 0$ in the reduced system. Note that $\mathcal{B}_{\tilde{C}}$ is a compact set and that the virtual capacities $\left\{\tilde{C}_{l}\right\}$ that lead to the system optimal rates (i.e., the solution to $\operatorname{SYSTEM}(U, S, C))$ lies in the interior of $\mathcal{B}_{\tilde{C}}$. Therefore, to prevent $\tilde{C}$ from leaving $\mathcal{B}_{\tilde{C}}$ in the original model, we set $\dot{\tilde{C}}_{l}=0$, whenever $\tilde{C}_{l}=n_{l} \hat{C}_{l}$ and $\lambda_{l}<C_{l}$.

From Section III, we know that the system is globally asymptotically stable when all the links are fully utilized in the solution to the original constrained optimization problem $\operatorname{SYSTEM}(U, S, C)$. We will now show that under 
Assumption 3, the reduced-system (17) is semiglobally exponentially stable. Appealing to Lemma IV.2, we require $\tilde{C}(0)$ to lie in $\mathcal{B}_{\tilde{C}}$. We rewrite (17) as

$$
\begin{aligned}
\dot{\lambda}^{*} & =\Lambda B^{-1}\left(C-\lambda^{*}\right) \\
& =-\beta S^{T} A^{-1} S B B^{-1}\left(C-\lambda^{*}\right) \\
& =-\beta S^{T} A^{-1} S\left(C-\lambda^{*}\right) .
\end{aligned}
$$

Defining $C-\lambda^{*}=\phi$, we can rewrite (17) as

$$
\dot{\phi}=\beta S^{T} A^{-1} S \phi .
$$

The Lyapunov function (30) can now be rewritten in terms of $\phi$ as

$$
\mathcal{W}(\phi):=\phi^{T} \phi
$$

The following theorem shows that the reduced system is semiglobally exponentially stable.

Theorem IV.1: Under Assumption 3, the reduced system given by (17) is semiglobally exponentially stable.

Proof: See Appendix VII.E.

NOTE: We need Assumption 3 to show that there is an unique $\tilde{C}$ which solves the optimization problem. Therefore, we can now look at the problem from the $\lambda^{*}$ space and then convert it back into the $\tilde{C}$ space as there exists a bijective map from the $\lambda^{*}$ space to the $\tilde{C}$ space.

We know that the boundary-layer system is globally asymptotically stable [4]. We will now show that the boundary-layer system is locally exponentially stable uniformly in $\tilde{C}$.

Lemma IV.3: The boundary-layer system given by (18) is locally exponentially stable uniformly in $\{\tilde{C}\}$. That is, there exists a $r>0$, such that

$$
\left\|x(t,\|x(0)\|, \tilde{C})-x^{*}(\tilde{C})\right\| \leq K\left\|x(0)-x^{*}(\tilde{C})\right\| \exp [-\gamma t]
$$

for all $(x, \tilde{C}) \in\left\{\left\|x-x^{*}(\tilde{C})\right\| \leq r\right\} \times \mathcal{B}_{\tilde{C}}$.

Proof: Linearizing the boundary-layer system (18) around the equilibrium point we get

$$
\Delta \dot{x}=C A \Delta x
$$

where $A$ is the Hessian matrix defined in (35) and $\mathrm{C}$ is the diagonal matrix defined as

$$
C:=\left[\begin{array}{cccc}
\frac{1}{U_{1}^{\prime}\left(x_{1}^{*}\right)} & 0 & \cdots & 0 \\
0 & \frac{1}{U_{2}^{\prime}\left(x_{2}^{*}\right)} & \cdots & 0 \\
\vdots & \vdots & \cdots & 0 \\
0 & 0 & \cdots & \frac{1}{U_{N}^{\prime}\left(x_{N}^{*}\right)}
\end{array}\right]
$$

Since $C>0$, all the eigenvalues of $C A$ are negative. Therefore, the system is locally exponentially stable. Let $\rho_{\max }(C A)<0$ be the maximum eigenvalue of $C A$. We know that the eigenvalues are continuous functions of the elements of $C A$, the elements of $C A$ are continuous functions of $\tilde{C}$ and $\tilde{C}$ lies in a compact set $\mathcal{B}_{\tilde{C}}$. Therefore, $\max _{\tilde{C}} \rho_{\max }(C A)<0$, and hence, $C A$ is uniformly Hurwitz in $\tilde{C}$. Moreover, the elements of $C A$ are continuously differentiable functions of $\tilde{C}$. Therefore, using [10, Lemma 5.12], we can conclude that there exists a $r>0$ such that the system is locally exponentially stable uniformly in $\tilde{C}$, for all $(x, \tilde{C}) \in\left\{\left\|x-x^{*}(\tilde{C})\right\|<r\right\} \times \mathcal{B}_{\tilde{C}}$.

Lemma IV.3 shows that the boundary-layer system is locally exponentially stable uniformly in $\tilde{C}$. We can now show that the boundary-layer system is semiglobally exponentially stable. i.e., if the user rates are constrained to lie in any compact set $\mathcal{B}_{x}$, then the boundary-layer system is exponentially stable uniformly in $\tilde{C}$ for all $\|x(0)\| \in \mathcal{B}_{x}$. Note that we require $\mathcal{B}_{x}$ to contain the set $\left\{x^{*}(\tilde{C}): C \in \mathcal{B}_{\tilde{C}}\right\}$. Since $\mathcal{B}_{\tilde{C}}$ is a compact set and $x^{*}(\tilde{C})$ is a continuously differentiable function of $\tilde{C}$, the set $\left\{x^{*}(\tilde{C}): \tilde{C} \in \mathcal{B}_{\tilde{C}}\right\}$ is also compact.

Theorem IV.2: The boundary-layer system given by (18) is semiglobally exponentially stable uniformly in $\{\tilde{C}\}$.

Proof: See Appendix VII.F

We now state the main result of the paper.

Theorem IV.3: Suppose the initial conditions $x(0)$ and $\tilde{C}(0)$ of the system lie in a compact set. Then, there exists an $\alpha$ small enough such that the singularly perturbed system (45)-(46) is exponentially stable. Moreover, the unique value $\left\{\bar{\lambda}_{l}\right\}$ at the stable point of the system solves $\operatorname{SYSTEM}(U, S, C)$.

Proof: See Appendix VII.G.

\section{CONCLUSION}

In this paper, we have shown that the shadow prices (Lagrange multipliers) of each link can be communicated to the end hosts even in networks where each node may use a different marking algorithm. This is achieved by adapting the marking probabilities (i.e., the fraction of marked packets in a fluid model) to attempt to drive the link utilization to a desired level. Even if a link is underutilized in the optimal fluid resource allocation, this scheme succeeds in conveying the correct information to the end hosts. We have also shown that the entire system comprising of congestion-controllers at the sources and the link adaptation scheme at the links is globally exponentially stable under some assumptions. This is achieved by appealing to the singular-perturbation theory and decomposing the system into two systems, a slow system (or the reduced system) and a fast system (or boundary-layer system). It is then shown that both the reduced system and the boundary-layer system are exponentially stable. Moreover, the boundary-layer system is shown to be exponentially stable uniformly with respect to the virtual capacity. We then show that the entire system is globally exponentially stable.

We have shown the stability of the entire system assuming that each link has a flow that passes only through it. While this is a limitation, we can approximate any system using this model by defining a flow through each link which has a utility function equal to a strictly increasing concave function multiplied by an arbitrarily small weighting factor. An open problem is to show that the stability of a such an approximation implies the stability of the original system.

In a real network, in addition to controllable flows, there would be uncontrollable flows such as very short file transfers. These would act as stochastic disturbances to the congestion-controlled long flows. We have studied this phenomenon using simulations. A challenging open question is to design a framework to incorporate these effects in the design of socially 
optimal, loss-free, delay-free networks. A practical solution to this problem is to design the adaptation algorithm to drive each link's utilization to a value smaller than 1 [6]. A mathematical model to drive the choice of this utilization would be desirable.

In practice, the step-size parameter $\alpha$ has to be chosen as a function of the round-trip propagation delays of the users to achieve stability. We have studied the problem of designing $\alpha$ to ensure stability in [13].

\section{APPENDIX I}

\section{A. Proof of Lemma III.1}

Let us consider link $l$. Let the solution to the single link optimization problem (19) be $\left(\bar{y}_{r}: l \in r\right)$. It can be easily seen that $\sum_{r: l \in r} \bar{y}_{r}=C_{l}$. Writing down the first-order necessary conditions for the Lagrangian, we get

$$
C_{l}=\sum_{r: l \in r} U_{r}^{\prime-1}\left[\frac{\nu_{l}}{\Delta_{r}}\right] .
$$

Comparing (4) and (51), we can see that

$$
\nu_{l}=\beta p_{l}\left(C_{l}, \tilde{C}_{l}^{(d)}\right)
$$

\section{B. Proof of Lemma III.2}

From (24), we know that $\mu_{l}>0$ only if $\bar{\lambda}_{l}=C_{l}$. Therefore, for each $l \in \mathcal{L}_{o}^{c}$, the condition $\mu_{l} \leq \nu_{l}$ holds trivially from (23) and (24). Let link $l$ be a congested link, i.e., $l \in \mathcal{L}_{O}$ Therefore, we know $\mu_{l}>0$. Assume that the claim is false, i.e., $\mu_{l}>\nu_{l}$. We know from (25)

$$
\begin{aligned}
C_{l} & =\sum_{r: l \in r} U_{r}^{\prime-1}\left[\sum_{j: j \in r} \frac{\mu_{j}}{\Delta_{r}}\right] \\
& =\sum_{r: l \in r} U_{r}^{\prime-1}\left[\frac{\mu_{l}}{\Delta_{r}}+\sum_{j: j \in r, j \neq l} \frac{\mu_{j}}{\Delta_{r}}\right] .
\end{aligned}
$$

However, $\mu_{j} \geq 0$ for all $j \in \mathcal{L}$. Therefore

$$
\mu_{l}+\sum_{j: j \in r, j \neq l} \mu_{j}>\nu_{l}
$$

Since, $U_{r}^{\prime-1}$ is a strictly decreasing function (from our assumptions on $\left.U_{r}\left(x_{r}\right)\right)$ for all $r \in \mathcal{R}$ and $\Delta_{r}>0$ for all $r \in \mathcal{R}$, we get

$$
\begin{aligned}
C_{l} & =\sum_{r: l \in r} U_{r}^{\prime-1}\left[\frac{\mu_{l}}{\Delta_{r}}+\sum_{j: j \in r, j \neq l} \frac{\mu_{j}}{\Delta_{r}}\right] \\
& <\sum_{r: l \in r} U_{r}^{\prime-1}\left[\frac{\nu_{l}}{\Delta_{r}}\right]=C_{l}
\end{aligned}
$$

which is a contradiction. Hence, $\mu_{l} \leq \nu_{l}$ for all $l \in \mathcal{L}$.

\section{Proof of Lemma IV.1}

From (40), we get

$$
\Omega=A^{-1} D
$$

Since, the derivative exists at each point, $x^{*}(\tilde{C})$ is a continuous function. Also, each element of $A^{-1}$ and $D$ are continuous functions of $\tilde{C}$. Hence, $x^{*}(\tilde{C})$ is a continuously differentiable function of $\tilde{C}$.

\section{Proof of Lemma IV.2}

Let us suppose that each link has at least one flow that is common to two or more links. If not, then the link is isolated from the rest of the network and this case can be handled easily. We need to show that $\tilde{C}_{l} \geq \hat{C}_{l}$ implies $\lambda_{l}^{*}>C_{l}$. Assume not, i.e, $\tilde{C}_{l} \geq \hat{C}_{l}$ and $\lambda_{l}^{*} \leq C_{l}$. Therefore, $x_{j}^{*}<C_{l}$ which implies $\Delta_{j} U_{j}^{\prime}\left(x_{j}^{*}\right)>\Delta_{j} U_{j}^{\prime}\left(\bar{C}_{l}\right)$. We know that

$$
\beta p_{l}\left(\lambda_{l}^{*}, \tilde{C}_{l}\right)=\Delta_{j} U_{j}^{\prime}\left(x_{j}^{*}\right)
$$

and from Assumption 1 that

$$
\beta p_{l}\left(\lambda_{l}^{*}, \hat{C}_{l}\right) \geq \beta p_{l}\left(\lambda_{l}^{*}, \tilde{C}_{l}\right) .
$$

We can now write

$$
\begin{aligned}
\beta p_{l}\left(\lambda_{l}^{*}, \hat{C}_{l}\right) & \geq \Delta_{j} U_{j}^{\prime}\left(x_{j}^{*}\right) \\
\Rightarrow \beta p_{l}\left(\lambda_{l}^{*}, \hat{C}_{l}\right) & >\Delta_{j} U_{j}^{\prime}\left(C_{l}\right) \\
\Rightarrow \beta p_{l}\left(\lambda_{l}^{*}, \hat{C}_{l}\right) & >\beta p_{l}\left(C_{l}, \hat{C}_{l}\right) \\
\Rightarrow \lambda_{l}^{*} & >C_{l}
\end{aligned}
$$

which is a contradiction. Therefore, $\dot{\widetilde{C}}_{l}<0$, whenever $\tilde{C}_{l} \geq \hat{C}_{l}$. Since, the reduced-system is globally exponentially stable, there exists a time $\hat{t}<\infty$, such that for all $t>\hat{t}, \tilde{C}_{l}(t)<n_{l} \hat{C}_{l}$. Similarly, we can show using Theorem III.1 that $\tilde{C}_{l}$ in the reduced system is always greater than or equal to zero. Therefore, $\tilde{C}_{l}$ in the reduced system eventually belongs to $\left[0, \hat{C}_{l}\right]$.

\section{E. Proof of Theorem IV.I}

Proof: Consider the Lyapunov function given by (49). We note that

$$
\frac{\partial \mathcal{W}}{\partial \phi} \dot{\phi}=2 \beta \phi^{T} S^{T} A^{-1} S \phi=2 \beta(S \phi)^{T} A^{-1}(S \phi) .
$$

We know that $A^{-1}(\tilde{C})$ is negative-definite for any value of $\tilde{C}$ and the elements of $A^{-1}(\tilde{C})$ are continuous in $\tilde{C}$. Also, by Assumption 3, since $S$ is a full-rank matrix, $S^{T} A^{-1} S$ is negative-definite. We also know that the eigenvalues of $S^{T} A^{-1} S$ are continuous functions of its elements, and therefore, continuous functions of $\tilde{C}$. Since, $\tilde{C}$ lies in a compact set, the absolute value of the maximum eigenvalue of $S^{T} A^{-1} S$ can be bounded by a constant $\sigma>0$. Therefore

$$
\frac{\partial \mathcal{W}}{\partial \phi} \dot{\phi} \leq-2 \beta \sigma\|\phi\|^{2}<0 .
$$


Hence, from [10, Cor. 3.4], (48) is globally exponentially stable.

We will now show that there exists an unique $\tilde{C}$ for every $\lambda^{*}$. By definition, for every $\lambda^{*}$, there exists an unique $x^{*}$, where $x$ is the vector of source rates. For each link $l \in \mathcal{L}$, consider a user that flows only through that link and denote that user by $l_{s}$. Assumption 3 guarantees the existence of such a user. Now

$$
\begin{aligned}
\Delta_{l_{s}} U_{l_{s}}^{\prime}\left(x_{l_{s}}^{*}\right) & =\beta p_{l}\left(\lambda_{l}^{*}, \tilde{C}_{l}\right), \quad \forall l \in \mathcal{L} \quad \text { and } \\
\lambda_{l}^{*} & =\sum_{r: l \in r}\left(\sum_{k: k \in r} \frac{\beta}{\Delta_{r}} p_{k}\left(\lambda_{k}^{*}, \tilde{C}_{k}\right)\right) \quad \forall l \in \mathcal{L} \\
\Rightarrow \lambda_{l}^{*} & =\sum_{r: l \in r}\left(\sum_{k: k \in r} \frac{\Delta_{k_{s}}}{\Delta_{r}} U_{k_{s}}^{\prime}\left(x_{k_{s}}^{*}\right)\right) \quad \forall l \in \mathcal{L} .
\end{aligned}
$$

Let $\left(y_{1}^{*}, y_{2}^{*}, \ldots, y_{N}^{*}\right)$ be the set of values of the flows that satisfies (16). Therefore

$$
p_{l}\left(\sum_{r: l \in r} \sum_{k: k \in r} \frac{\Delta_{k_{s}}}{\Delta_{r}} U_{k_{s}}^{\prime}\left(y_{k_{s}}^{*}\right), \tilde{C}_{l}\right)=\frac{\Delta_{l_{s}}}{\beta} U_{l_{s}}^{\prime}\left(y_{l_{s}}^{*}\right) .
$$

Since, for each $s$, there exists an unique value of $q$ satisfying the equation $p(s, q)=$ constant, there exists an unique $\tilde{C}$, that leads to $x^{*}=\left(y_{1}^{*}, y_{2}^{*}, \ldots, y_{N}^{*}\right)$. Also, $\lambda^{*}$ itself is a continuously differentiable function of $\widetilde{C}$, i.e., $\lambda^{*}=h(\tilde{C})$, and hence, $h^{-1}$ exists and is continuously differentiable. By the mean value theorem, we have

$$
\tilde{C}=h^{-1}(C)+\left.\frac{d h^{-1}\left(\lambda^{*}\right)}{d \lambda^{*}}\right|_{\lambda^{*}=y_{1}}\left(\lambda^{*}-C\right)
$$

where $\lambda^{*}=C$ is the equilibrium point and $y_{1}=\epsilon_{1} C+(1-$ $\left.\epsilon_{1}\right) \lambda^{*}$, for some $0<\epsilon_{1}<1$. Let $\widetilde{C}^{*}$ be the virtual capacity at the stable/equilibrium point of the reduced-system. Therefore, we have $\tilde{C}^{*}=h^{-1}(C)$. Hence

$$
\left\|\tilde{C}-\tilde{C}^{*}\right\| \leq\left\|\left.\frac{d h^{-1}\left(\lambda^{*}\right)}{d \lambda^{*}}\right|_{\lambda^{*}=y_{1}}\right\|\left\|\lambda^{*}-C\right\| .
$$

Let the system given by (48) have an initial condition, $\lambda^{*}(0)$. Since, the system given by (48) is exponentially stable, we have for some $k, \gamma>0$

$$
\begin{aligned}
& \left\|\lambda^{*}-C\right\| \leq k\left\|\lambda^{*}(0)-C\right\| \exp [-\gamma t] \\
& \Rightarrow\left\|\tilde{C}-\tilde{C}^{*}\right\| \leq\left\|\left.\frac{d h^{-1}\left(\lambda^{*}\right)}{d \lambda^{*}}\right|_{\lambda^{*}=y_{1}}\right\| k\left\|\lambda^{*}(0)-C\right\| \exp [-\gamma t] .
\end{aligned}
$$

Using the mean-value theorem again, we get

$$
\left\|\lambda^{*}(0)-C\right\| \leq\left\|\left.\frac{d h(\tilde{C})}{d \tilde{C}}\right|_{\tilde{C}=y_{2}}\right\|\left\|\tilde{C}(0)-\tilde{C}^{*}\right\|
$$

where $\tilde{C}(0)$ is the virtual capacity corresponding to the initial condition $\lambda^{*}(0)$ and $y_{2}=\epsilon_{2} \tilde{C}^{*}+\left(1-\epsilon_{2}\right) \tilde{C}(0)$, for some $0<$ $\epsilon_{2}<1$. Also, $h(\tilde{C})$ and $h^{-1}\left(\lambda^{*}\right)$ are continuously differentiable functions. Therefore, we have

$$
\begin{aligned}
\left\|\tilde{C}-\tilde{C}^{*}\right\| \leq k\left\|\left.\frac{d h^{-1}\left(\lambda^{*}\right)}{d \lambda^{*}} \frac{d h(\tilde{C})}{d \tilde{C}}\right|_{\substack{\tilde{C}=y_{2} \\
\lambda^{*}=y_{1}}}\right\| \\
\\
\quad\left\|\tilde{C}(0)-\tilde{C}^{*}\right\| \exp [-\gamma t], \\
\Rightarrow\left\|\tilde{C}-\tilde{C}^{*}\right\| \leq \hat{k}\left\|\tilde{C}(0)-\tilde{C}^{*}\right\| \exp [-\gamma t]
\end{aligned}
$$

where

$$
\hat{k}=k \max _{\lambda^{*}}\left\|\left.\frac{d h^{-1}\left(\lambda^{*}\right)}{d \lambda^{*}}\right|_{\lambda^{*}=y_{1}}\right\| \max _{\tilde{C}}\left\|\left.\frac{d h(\tilde{C})}{d \tilde{C}}\right|_{\tilde{C}=y_{2}}\right\|>0 .
$$

Hence, (17) is also exponentially stable.

\section{F. Proof of Theorem IV.2}

We know that the system is locally exponentially stable uniformly in $\tilde{C}$. That is, there exists a $r>0$ such that

$$
\begin{aligned}
& \|x(t,\|x(0)\|, \tilde{C})\| \leq K\|x(0)\| \exp ^{-\gamma t}, \\
& \forall \tilde{C} \in \mathcal{B}_{\tilde{C}},\|x(0)\| \leq r
\end{aligned}
$$

where $K>1$ and $\gamma>0$ are constants independent of $\tilde{C}$ and $\mathcal{B}_{\tilde{C}}$ is a compact set. Let us choose $r<1$ without loss of generality. Note: When we say $\|x\|$ we mean $\left\|x-x^{*}\right\|$. Also, from the continuous dependence of the solution on parameters and initial conditions, we know that $\|x(t,\|x(0)\|, \tilde{C})\|$ is a continuous function of $t,\|x(0)\|$, and $\mathscr{C}$. Define

$$
T^{r}(\|x(0)\|, \tilde{C}):=\inf \{s>0:\|x(s,\|x(0)\|, \tilde{C})\| \leq r\} .
$$

We know that

- $T^{r}(\|x(0)\|, \tilde{C})<\infty$ for each $\|x(0)\|$ and $\tilde{C}$;

- $T^{r}(\|x(0)\|, \tilde{C})$ is a continuous function of $\|x(0)\|$ and $\tilde{C}$ as $x(t,\|x(0)\|, \widetilde{C})$ is a continuous function of $t,\|x(0)\|$ and $\tilde{C}$.

Therefore, let

$$
T_{\max }^{r}:=\max _{\|x(0)\| \in B_{x}, \tilde{C} \in \mathcal{B}_{\tilde{C}}} T^{r}(\|x(0)\|, \tilde{C})
$$

where $B_{x}$ is a compact set in which $\|x(0)\|$ resides. Now, $T_{\max }^{r}<\infty$ and is well defined. Now, define

$$
\begin{gathered}
M:=\max _{\substack{t \in\left[0, T_{\max }^{r}\right]\\
}} \| x\left(t,\|x(0)\| \in B_{x}\right. \\
\\
\tilde{C} \in \mathcal{B}_{\tilde{C}}
\end{gathered}
$$

Note that, we are maximizing a continuous function over compact sets and as a results $M$ exists and $M<\infty$. Define a new function

$$
\Theta(t):= \begin{cases}M & 0 \leq t \leq T_{\max }^{r} \\ 0 & \text { otherwise. }\end{cases}
$$

Define

$$
\Sigma(t):=M+\gamma T_{\max }^{r}-\gamma t
$$


Let

$$
\tilde{T}=\inf \{s>0: \Sigma(t) \leq r\}
$$

It is clear that $\tilde{T}<\infty$. We can now rewrite $\Sigma(t)$ as:

$$
\Sigma(t)=r+\gamma \tilde{T}-\gamma t
$$

Now,

$$
\Sigma(t) \geq \Theta(t) \quad \forall t \leq \tilde{T}
$$

We now make the following claims.

Claim 1:

$$
\|x(t,\|x(0)\|, \tilde{C})\| \leq \Sigma(t) \quad \forall t \in[0, \tilde{T}]
$$

Proof: We know

$$
\|x(t,\|x(0)\|, \tilde{C})\| \leq \Theta(t) \leq \Sigma(t) \quad \forall t \in\left[0, T_{\max }^{r}\right],
$$

and

$$
\|x(t,\|x(0)\|, \tilde{C})\| \leq r \leq \Sigma(t) \quad \forall t \in\left[T_{\max }^{r}, \tilde{T}\right] .
$$

Hence, it is proved.

Define

$$
L:=\frac{K \exp [\gamma \tilde{T}]}{r} .
$$

Claim 2: The system is exponentially stable in the region $B_{x} \times \mathcal{B}_{\tilde{C}}$. That is

$$
\|x(t,\|x(0)\|, \tilde{C})\| \leq L\|x(0)\| \exp [-\gamma t]
$$

Proof: Case 1): $\|x(0)\|>r$. In this case, let us consider the system in the time interval $[0, \tilde{T}]$. Note that $K>1, r<1$ and $(\|x(0)\| / r)>1$.

$$
\begin{aligned}
L\|x(0)\| \exp [-\gamma t] & =K \exp [\gamma \tilde{T}] \frac{\|x(0)\|}{r} \exp [-\gamma t] \\
& \geq \exp (\gamma \tilde{T}) \exp [-\gamma t] \\
& =\exp [-\gamma(t-\tilde{T})] \\
& \geq 1-\gamma(t-\tilde{T}) \\
& =1+\gamma \tilde{T}-\gamma t \\
& \geq r+\gamma \tilde{T}-\gamma t \\
& =\Sigma(t) \\
& \geq\|x(t,\|x(0)\|, \tilde{C})\| .
\end{aligned}
$$

Therefore, for all $t \leq \tilde{T}$, we have

$$
\|x(t,\|x(0)\|, \tilde{C})\| \leq L\|x(0)\| \exp [-\gamma t]
$$

For $t>\tilde{T}$, we know that $\|x(t,\|x(0)\|, \tilde{C})\|<r$, and therefore, we can use case 2$)$ setting $\|x(0)\|=\|x(\tilde{T},\|x(0)\|, \tilde{C})\|$.

Case 2): $\|x(0)\| \leq r$. In this case, we know that the system is exponentially stable. Also, we can take $\tilde{T}=0$ in this case.
Therefore, we need to show that $(K / r) \geq K$, which is trivially satisfied as $r<1$.

Therefore, the system is exponentially stable in the region $B_{x} \times \mathcal{B}_{\tilde{C}}$. Hence, it is proved.

\section{G. Proof of Theorem IV.3}

Define

$$
g_{l}^{e}(x, \tilde{C}):=C_{l}-\sum_{r: l \in r} x_{r} .
$$

Let $g^{e}(x, \tilde{C})=\left[g_{1}^{e}(x, \tilde{C}), \ldots, g_{M}^{e}(x, \tilde{C})\right]^{\prime}$, where $M$ is the number of links in the networks. We know that $g^{e}(x, \tilde{C})$ is a continuously differentiable function of $x$ and $\tilde{C}$. The reducedsystem is given by

$$
\dot{\tilde{C}}_{l}:=g_{l}^{r}(\tilde{C})=C_{l}-\sum_{r: l \in r} x_{r}^{*}(\tilde{C})
$$

where $x^{*}(\tilde{C})$ is the unique root to the equation $f(x, \tilde{C})=0$. We know that

- $f, g^{e}$ and $g^{r}$ are continuously differentiable functions from our assumptions on the utility functions and marking functions and from Lemma IV.1;

- the reduced system is semiglobally exponentially stable, as shown in Theorem IV.1;

- the boundary-layer system is semiglobally exponentially stable uniformly in $\tilde{C}$ as shown in Theorem IV.2.

If we assume that the virtual capacities can take negative values i.e., $\tilde{C} \in \mathcal{R}$, then we can apply [14, Lemma 1] to infer semiglobal exponential stability. However, since we restrict the virtual capacities to be positive, i.e., $\tilde{C} \in \mathcal{R}^{+}$, we need to show that the Lyapunov function is negative-definite at the boundary points where $\tilde{C}_{i}=0$ and $\lambda_{i}>C_{i}$. Assume that the initial conditions $x(0)$ and $\tilde{C}(0)$ belongs to $B_{x(0)}$ and $B_{\tilde{C}(0)}$ respectively, where both $B_{x(0)}$ and $B_{\tilde{C}(0)}$ are compact sets. Let $\mathcal{H}(t, \tilde{C})$ be the trajectory of the reduced-system starting at $\tilde{C}$ at time $t$ and let $\mathcal{S}(\tau, x ; \tilde{C})$ be the trajectory of the boundary-layer system starting at the initial point $x$ at time $\tau=0$. We know that the reduced system is exponentially stable whenever $\tilde{C}(0) \in B_{\tilde{C}(0)}$ from Theorem IV.1. From Theorem IV.2, the boundary-layer system is exponentially stable uniformly in $\tilde{C}$ whenever $x(0)$ and $\tilde{C}$ are constrained to lie in a compact set. Define $\tilde{C}^{*}$ to be the virtual capacity that solves $\operatorname{SYSTEM}(U, S, C)$. As defined in [15], consider the conceptual Lyapunov functions

$$
\begin{aligned}
\mathcal{V}(\tilde{C}) & :=\int_{0}^{T}\left\|\mathcal{H}(t, \tilde{C})-\tilde{C}^{*}\right\|^{2} d t \\
\mathcal{W}(x, \tilde{C}) & :=\int_{0}^{T}\left\|\mathcal{S}(\tau, x ; \tilde{C})-x^{*}(\tilde{C})\right\|^{2} d \tau,
\end{aligned}
$$

where $T$ is a constant defined in [15]. We know that $\mathcal{V}(\tilde{C})$ and $\mathcal{W}(x, \tilde{C})$ are positive-definite radially unbounded functions. Let

$$
K_{1}:=\max _{\tilde{C} \in B_{\tilde{C}(0)}} \mathcal{V}(\tilde{C}),
$$

and

$$
K_{2}:=\max _{x \in B_{x(0)} ; \tilde{C} \subset B_{\tilde{C}(0)}} \mathcal{W}(x, \tilde{C})
$$


We know that both $K_{1}$ and $K_{2}$ exists and are finite. Fix $0<d<$ 1. Define

and

$$
\Gamma_{\tilde{C}}:=\left\{\tilde{C} \geq 0: \mathcal{V}(\tilde{C}) \leq \frac{K_{1}+K_{2}}{d}\right\}
$$

$$
\Gamma_{x}:=\left\{x: \mathcal{W}(x, \tilde{C}) \leq \frac{K_{1}+K_{2}}{1-d}, \quad \forall \tilde{C} \in \Gamma_{\tilde{C}}\right\}
$$

We can easily show that both $\Gamma_{\tilde{C}}$ and $\Gamma_{x}$ are compact sets. Also, without loss of generality, we can assume that the marking functions $p_{l}(r, s)$ is differentiable with respect to $s$ at $s=0$. From [14], we can show that $\mathcal{V}(\tilde{C})$ and $\mathcal{W}(x, \tilde{C})$ are Lyapunov functions for the reduced system and the boundary-layer systems respectively in the compact set $\Gamma_{x} \times \Gamma_{\tilde{C}}$ when the initial conditions are constrained to lie in $B_{x(0)} \times B_{\tilde{C}(0)}$. It also follows from [14], that there exists positive constants $e_{1}, e_{2}, \gamma_{1}$, and $\gamma_{2}$ such that

$$
\begin{aligned}
\left(\nabla_{\tilde{C}} \mathcal{V}(\tilde{C})\right)^{\prime} g^{r}(\tilde{C}) \leq & -\gamma_{1}\left\|\tilde{C}-\tilde{C}^{*}\right\|^{2}, \quad \forall \tilde{C} \in \Gamma_{\tilde{C}} \\
\left\|\nabla_{\tilde{C}} \mathcal{V}(\tilde{C})\right\| \leq & e_{1}\left\|\tilde{C}-\tilde{C}^{*}\right\|, \quad \forall \tilde{C} \in \Gamma_{\tilde{C}} \\
\left(\nabla_{x} \mathcal{W}(x, \tilde{C})\right)^{\prime} f(x, \tilde{C}) \leq & -\gamma_{2}\left\|x-x^{*}(\tilde{C})\right\|^{2} \\
& \times \forall(x, \tilde{C}) \in \Gamma_{x} \times \Gamma_{\tilde{C}}
\end{aligned}
$$

and

$$
\left\|\nabla_{x} \mathcal{W}(x, \tilde{C})\right\| \leq e_{2}\left\|x-x^{*}(\tilde{C})\right\|, \quad \forall(x, \tilde{C}) \in \Gamma_{x} \times \Gamma_{\tilde{C}} . \text { Hence }
$$

Define the Lyapunov function $\nu(x, \tilde{C})$ for the original singularly perturbed system as

$$
\nu(x, \tilde{C}):=d \mathcal{V}(\tilde{C})+(1-d) \mathcal{W}(x, \tilde{C}) .
$$

We can easily show that

$$
\left\{(x, \tilde{C}): \tilde{C} \geq 0 \quad \text { and } \quad \nu(x, \tilde{C}) \leq K_{1}+K_{2}\right\} \subseteq \Gamma_{x} \times \Gamma_{\tilde{C}}
$$

We now know that

$$
\nu(x(0), \tilde{C}(0))<K_{1}+K_{2} .
$$

Also, the equation shown at the bottom of the page holds true. Define the set $\Xi$ as

$\Xi=\left\{(x, \tilde{C}) \in \Gamma_{x} \times \Gamma_{\tilde{C}}: \tilde{C}_{i}=0\right.$ and $g_{i}(x)=0$ for some $\left.i\right\}$.

Clearly, $\Xi$ is a compact set. Note that, $g(x)=g^{e}(x)$ for all $(x, \tilde{C}) \in \Gamma_{x} \times \Gamma_{\tilde{C}} \backslash \Xi$ and $\left\|g^{e}(x)\right\| \geq\|g(x)\|$ for all $(x, \tilde{C}) \in \Xi$. Also, note that the equilibrium point $\left(x^{*}, \tilde{C}^{*}\right) \in\left(\Gamma_{x} \times \Gamma_{\tilde{C}}\right) \backslash$ $\Xi$. Since, $\Gamma_{x} \times \Gamma_{\tilde{C}}$ is a compact set and $f, g^{e}$ and $x^{*}(\tilde{C})$ are continuously differentiable functions, we have from [14]

- for all $(x, \tilde{C}) \in\left(\Gamma_{x} \times \Gamma_{\tilde{C}}\right) \backslash \Xi$

$$
\left\|g(x, \tilde{C})-g\left(x^{*}(\tilde{C}), \tilde{C}\right)\right\| \leq c_{1}\left\|x-x^{*}(\tilde{C})\right\| ;
$$

- for all $(x, \tilde{C}) \in\left(\Gamma_{x} \times \Gamma_{\tilde{C}}\right) \backslash \Xi$,

$$
\begin{aligned}
\left(\nabla_{\tilde{C}} \mathcal{V}(\tilde{C})\right)^{\prime}\left[g(x, \tilde{C})-g\left(x^{*}(\tilde{C})\right.\right. & , \tilde{C})] \\
& \leq c_{2}\left\|\tilde{C}-\tilde{C}^{*}\right\|\left\|x-x^{*}(\tilde{C})\right\|
\end{aligned}
$$

- for all $\forall(x, \tilde{C}) \in\left(\Gamma_{x} \times \Gamma_{\tilde{C}}\right) \backslash \Xi$,

$\left(\nabla_{\tilde{C}} \mathcal{W}(x, \tilde{C})\right)^{\prime} g(x, \tilde{C})$

$$
\leq c_{3}\left\|x-x^{*}(\tilde{C})\right\|^{2}+c_{4}\left\|\tilde{C}-\tilde{C}^{*}\right\|\left\|x-x^{*}(\tilde{C})\right\|
$$

where the $c_{i}$ 's are positive constants. Now, for all $(x, \tilde{C}) \in$ $\Xi, g_{i}(x, \tilde{C})=0$ for some $i$. Let $\Upsilon$ be defined as

$$
\Upsilon(x, \tilde{C}):=\left\{i: \tilde{C}_{i}=0, g_{i}(x, \tilde{C})=0\right\} \quad \forall(x, \tilde{C}) \in \Xi .
$$

However, $g_{i}\left(x^{*}(\tilde{C}), \tilde{C}\right)>0$ and $g_{i}^{e}(x, \tilde{C})<0$ for all $i \in \Upsilon$. Also, $g_{i}^{e}\left(x^{*}(\tilde{C}), \tilde{C}\right)=g_{i}\left(x^{*}(\tilde{C}), \tilde{C}\right)=g_{r}(\tilde{C})$. Therefore

$$
\begin{aligned}
& \left\|g_{i}(x, \tilde{C})-g_{i}\left(x^{*}(\tilde{C}), \tilde{C}\right)\right\| \\
& \quad \leq\left\|g_{i}^{e}(x, \tilde{C})-g_{i}^{e}\left(x^{*}(\tilde{C}), \tilde{C}\right)\right\|, \quad \forall i \in \Upsilon
\end{aligned}
$$

and

$$
\begin{aligned}
& \left\|g_{i}(x, \tilde{C})-g_{i}\left(x^{*}(\tilde{C}), \tilde{C}\right)\right\| \\
& \quad=\left\|g_{i}^{e}(x, \tilde{C})-g_{i}^{e}\left(x^{*}(\tilde{C}), \tilde{C}\right)\right\| \quad \forall i \notin \Upsilon .
\end{aligned}
$$

$\left\|g(x, \tilde{C})-g\left(x^{*}(\tilde{C}), \tilde{C}\right)\right\| \leq c_{1}\left\|x-x^{*}(\tilde{C})\right\| \quad \forall(x, \tilde{C}) \in \Xi$

Therefore, we can write: $\forall(x, \tilde{C}) \in \Xi$

$$
\begin{aligned}
\left(\nabla_{\tilde{C}} \mathcal{V}(\tilde{C})\right)^{\prime} & {\left[g(x, \tilde{C})-g\left(x^{*}(\tilde{C}), \tilde{C}\right)\right] } \\
& \leq c_{2}\left\|\tilde{C}-\tilde{C}^{*}\right\|\left\|x-x^{*}(\tilde{C})\right\|
\end{aligned}
$$

Consider,

$$
\begin{aligned}
\left(\nabla_{\tilde{C}} \mathcal{W}(x, \tilde{C})\right)^{\prime} g(x, \tilde{C})= & 2 \int_{0}^{T}\left[S(\tau, x ; \tilde{C})-x^{*}(\tilde{C})\right]^{\prime} \\
& \times\left[\nabla_{\tilde{C}}\left(S(\tau, x ; \tilde{C})-x^{*}(\tilde{C})\right)\right] g(x, \tilde{C})
\end{aligned}
$$

We can also easily show that [14] $\nabla_{\tilde{C}_{i}}(S-h)$ is uniformly bounded. Therefore, we can write [14]

$$
\begin{aligned}
& \left(\nabla_{\tilde{C}} \mathcal{W}(x, \tilde{C})\right)^{\prime} g(x, \tilde{C}) \\
& \quad \leq c_{3}\left\|x-x^{*}(\tilde{C})\right\|^{2}+c_{4}\left\|\tilde{C}-\tilde{C}^{*}\right\|\left\|x-x^{*}(\tilde{C})\right\|
\end{aligned}
$$

As a result, from [14, Th. 1], we can conclude that there exists an $\alpha$ small enough such that

$$
\dot{\nu}(x, \tilde{C})<0 .
$$

Therefore, we can conclude that when the initial conditions are restricted to a compact set, the system is exponentially stable for

$$
\dot{\nu}(x, \tilde{C})=\left\{\begin{array}{l}
(1-d)\left(\nabla_{\tilde{C}} \mathcal{V}(\tilde{C})\right)^{\prime} g(x) \\
\quad+\frac{d}{\alpha}\left(\nabla_{x} \mathcal{W}(x, \tilde{C})\right)^{\prime} \cdot f(x, \tilde{C})+d\left(\nabla_{\tilde{C}} \mathcal{W}(x, \tilde{C})\right)^{\prime} \cdot g(x)
\end{array}\right.
$$


small enough $\alpha$. The second part of the theorem follows directly from Theorem III.3.

\section{ACKNOWLEDGMENT}

The authors would like to thank A. Teel for his comments and suggestions on an earlier draft of this paper.

\section{REFERENCES}

[1] R. J. Gibbens and F. P. Kelly, "Resource pricing and the evolution of congestion control," Automatica, 1999.

[2] F. P. Kelly, P. Key, and S. Zachary, "Distributed admission control," IEEE J. Select. Areas Commun., vol. 18, pp. 2617-2628, Dec. 2000.

[3] K. K. Ramakrishnan and S. Floyd, "A proposal to add explicit congestion notification (ECN) to IP,", Jan. 1999. RFC 2481.

[4] F. P. Kelly, A. Maulloo, and D. Tan, "Rate control in communication networks: shadow prices, proportional fairness and stability," J. Oper. Res. Soc., vol. 49, pp. 237-252, 1998.

[5] S. H. Low and D. E. Lapsley, "Optimization flow control I: Basic algorithm and convergence," IEEE/ACM Trans. Networking, vol. 7, pp. 861-875, Dec. 1999.

[6] S. Kunniyur and R. Srikant, "End-to-end congestion control: Utility functions, random losses and ECN marks," presented at the INFOCOM 2000, Tel Aviv, Israel, Mar. 2000.

[7] S. Athuraliya, D. E. Lapsley, and S. H. Low, "Random early marking for Internet congestion control," Proc. IEEE GLOBECOM, 1999.

[8] D. D. Botvich and N. G. Duffield, "Large deviations, economies of scale, and the shape of the loss curve in large multiplexers," Queueing Syst., vol. 20, pp. 293-320, 1995

[9] D. Bertsekas, Nonlinear Programming. Belmont, MA: Athena Scientific, 1999.

[10] H. Khalil, Nonlinear Systems, 2nd ed. Upper Saddle River, NJ: Prentice-Hall, 1996.

[11] S. Kunniyur and R. Srikant, "A time-scale decomposition approach to adaptive ECN marking," presented at the INFOCOM 2001, Anchorage, Alaska, Apr. 2001.

[12] F. P. Kelly, "Charging and rate control for elastic traffic," Euro. Trans. Telecommun., vol. 8, pp. 33-37, 1997.

[13] S. Kunniyur and R. Srikant, "Analysis and design of an adaptive virtual queue (AVQ) algorithm for active queue management," presented at the Proc. SIGCOMM 2001, San Deigo, CA, Aug. 2001.
[14] A. Saberi and H. Khalil, "Quadratic-type Lyapunov functions for singularly perturbed systems," IEEE Trans. Automat. Contr., vol. AC-29, pp. 542-550, June 1984.

[15] N. N. Krasovskii, Stability of Motion. Stanford, CA: Stanford Univ. Press, 1963.

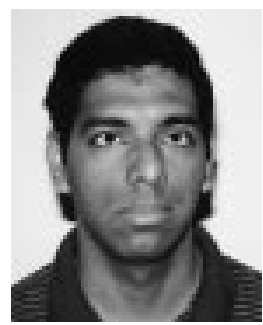

Srisankar Kunniyur received the B.E. degree in electrical and electronics engineering from Birla Institute of Technology and Science (B.I.T.S.), Pilani, India, and the M.S. and Ph.D. degrees in electrical engineering from the University of Illinois at Urbana-Champaign, in 1996, 1998, and 2001, respectively.

He is currently an Assistant Professor of Electrical Engineering at the University of Pennsylvania, Philadelphia. His research interests include design and performance analysis of high-speed communication networks, wireless and ad hoc networks, and congestion control and pricing in heterogeneous networks.

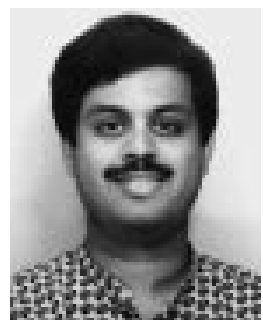

R. Srikant (S'90-M'91-SM'01) received the B.Tech. degree from the Indian Institute of Technology, Madras, and the M.S. and Ph.D. degrees from the University of Illinois, at Urbana-Champaign, in 1985, 1988, and 1991, respectively, all in electrical engineering.

He was a Member of the Technical Staff at AT\&T Bell Laboratories, Murray Hill, NJ, from 1991 to 1995. Since August 1995, he has been with the University of Illinois at Urbana-Champaign, where he is currently an Associate Professor in the Department of General Engineering and Coordinated Science Laboratory, and an Affiliate in the Department of Electrical and Computer Engineering. He is an Associate Editor of the IFAC journal Automatica. His research interests include communication networks, queueing theory, information theory, and stochastic control.

Dr. Srikant is the Chair of the 2002 IEEE Computer Communications Workshop, and an Editor of the IEEE/ACM TRANSACTIONS ON NETWORKING. He received an National Science Foundation CAREER award in 1997. 